\title{
Éditorial : Une nouvelle étape pour La Revue canadienne du vieillissement
}

Avec ce numéro de La Revue canadienne du vieillissement, j'entreprends ma deuxième année comme rédactrice en chef. C'est la première fois cependant que je m'adresse directement aux lecteurs par le biais d'un éditorial. Étant donné que de nombreux articles sont en attente de publication dans La Revue, j'avais choisi jusqu'à maintenant d'ajouter un article à plusieurs numéros plutôt que de consacrer un espace précieux à l'éditorial. Mais les circonstances viennent de faire prendre un nouveau virage à $L a$ Revue. En effet, l'appui renouvelé du Conseil de recherches en sciences humaines du Canada (CRSH) et une nouvelle subvention de l'Institut du vieillissement des Instituts de recherche en santé du Canada fait franchir à La Revue une nouvelle étape de son existence.

Je suis donc extrêmement fière d'annoncer que notre candidature à l'Aide aux revues de recherche et de transfert du CRSH a été retenue. On nous accorde en effet du financement pour une période additionnelle de trois ans, à compter d'avril 2002. C'est avec une immense gratitude que nous soulignons l'appui du CRSH. L'appui du Conseil depuis les débuts de $\mathrm{La}$ Revue de concert avec le financement de base de l'Association canadienne de gérontologie a permis que notre publication se mérite le respect et la crédibilité des divers milieux à qui elle s'adresse.

Je suis également très heureuse d'annoncer que notre candidature à l'Institut du vieillissement des Instituts de recherche en santé du Canada nous a mérité une subvention de trois ans. Au nom de toute l'équipe des rédacteurs/rédactrices, je tiens à remercier sincèrement le directeur de l'Institut, Réjean Hébert, ainsi que son comité consultatif de leur appui indéfectible. La subvention nous permettra d'augmenter la portée et la qualité de $\mathrm{La}$ Revue. Ce premier numéro du Volume 21 a fait peau neuve. Mais, plus important encore, il inaugure la nouvelle pratique qui consistera à publier beaucoup plus d'articles que par le passé. Ainsi, le temps entre l'acceptation d'un article et sa parution sera d'autant réduit. Je sais pertinemment que les délais de publication ont été source de frustration pour les auteurs, ce que je peux parfaitement comprendre, à un point tel qu'ils ont parfois découragé certains d'entre vous à soumettre leurs textes à $L a$ Revue dans les derniers mois. Nous pouvons vous assurer que la situation sera nettement améliorée et je peux prédire de façon presque certaine que d'ici peu, les auteurs n'auront plus à attendre plus de six ou huit mois avant que leur texte accepté ne soit publié.
Le nouveau financement nous permet d'ajouter de nouvelles sections à La Revue. À l'occasion, nous publierons des données sur des thèmes variés comme "Enjeux et commentaires", "Notes de recherche", "La pratique", "Le milieu clinique", etc. La Revue devrait faire paraître bientôt les directives de rédaction sur ces nouvelles sections. La section des comptes rendus sera plus étoffée et nous rédigerons des éditoriaux plus régulièrement qu'au cours des deux dernières années. Enfin, nous serons en mesure de publier promptement des articles et des rapports dont le sujet exige une diffusion rapide.

Ce numéro de La Revue présente une section "Enjeux et commentaires" où le professeur Benjamin Gottlieb se penche sur les circonstances et les difficultés reliées à la présence des bénévoles dans les organismes de services. La Revue en profite pour souligner l'importance de l'Année du bénévole qui vient de se terminer. Les questions soulevées par le professeur Gottlieb présentent une importance particulière en ces temps où, dans plusieurs provinces canadiennes, les politiques gouvernementales et les orientations de financement semblent reposer sur l'hypothèse que les bénévoles pourront et voudront effectuer le travail attribué normalement à des salariés et que les organismes auront les ressources nécessaires pour former les bénévoles et les intégrer à leurs modalités de prestation de services.

La Revue franchit véritablement un nouveau seuil qui lui offre l'occasion de prendre de l'expansion et de se diversifier, de rendre son contenu plus intéressant et d'en rehausser la qualité et d'emboîter le pas aux progrès de l'édition universitaire. Mais nos capacités devront être comblées par la soumission de textes rédigés par des chercheurs. Nous vous invitons donc à encourager vos collègues de Canada et d'ailleurs à présenter leurs travaux à $L a$ Revue et à participer du même coup à la nouvelle aventure de La Revue canadienne $d u$ vieillissement.

La rédactrice en chef

Carolyn J. Rosenthal 